\title{
The storage principle of DNA information
}

\author{
[Zhe Yin]
}

\begin{abstract}
In this paper, the author's stability theorem and the bridging property of hydrogen bonds are presented, and the formation and storage principle of DNA information are expounded by using the author's gravitational wave theorem. It has reference value for the research of DNA information, the stability of electromagnetic wave laser and the stability of quantum communication.
\end{abstract}

Key words---DNA information; gravitational wave; stability theorem

\section{Introduction}

All things in the world are moving, and if the two sub-parts of the same wave source (matter) are synchronous, they can be called relative static relations. There is no absolute stationary matter (or wave source).[1,2,4]

The stability theorem proposed in this paper is the relative stability law of wave source (matter) movement. (with unconstrained natural repairable property).

Rotating electrons, objects, planets and stars are called wave sources. The revolution and rotation are relative. If the reference object is not determined in the universe, all matter (wave source) rotates. Including single electron motion.

Wave source generates energy field. The universe is a relatively stable compound energy field. The energy field is the changing field (variable force field), and the intensity is different from the distance from the wave source. Wave source and energy field generate force, and force is the product of wave source.[1,6]

\section{Gravitational wave theory}

Gravitational waves existence theory [1,2,3,4,5,6,7]: There are P, Q two points. $\mathrm{P}$ is the wave source of gravitational field, and $\mathrm{Q}$ is a point in the gravitational field. The existence of energy rotational motion (including proton, neutron, atomic nucleus or planet) at $\mathrm{P}$ is the necessary and sufficient condition of the existence of gravitational waves at $\mathrm{Q}$. Direction is the bidirection of the path tangent at point $\mathrm{Q}$, and the limit of the convergence direction is $\mathrm{P}$ point.

Prove: Let say the distance of $\mathrm{P}$ and $\mathrm{Q}$ is $\mathrm{r}$, if $\mathrm{P}, \mathrm{Q}$ are stationary points, it only exists the physical factor of "distance $\mathrm{r}$ ", do not form elements of waves. Only the rotational movement can generate speed and energy.

That is the speed of $\mathrm{Q}$ point $V b$ and the angular velocity of $\mathrm{P}$ point $d \theta$ have a functional relationship.

$$
\begin{gathered}
V b=f(d \theta, r) \text {, when time } \Delta t \rightarrow 0 \text {, angular velocity } \Delta \theta \rightarrow 0 \text {, and } \\
\qquad b b=\frac{d r}{d t}=\lim _{\Delta \theta \rightarrow 0} \frac{[f(\theta+\Delta \theta), r]-[f(\theta), r]}{\Delta \theta},
\end{gathered}
$$

We have a conclusion that $\mathrm{P}$ is the wave source of gravitation, and point $\mathrm{Q}$ exists the gravitational wave emitted from source P. 


\section{Stability theorem}

If the increment of a function $f(x)$ satisfies the linear equation, this function is stable.

Rectangular coordinate system representation: $\mathbf{f}(\mathbf{x})$ is $n+1$ derivatives, $\mathrm{f}_{\mathrm{n}+1}(\mathrm{x})$ is the sum of the first $\mathrm{n}+1$ terms of Taylor's polynomial, and $\mathrm{R}_{\mathrm{n}}(\mathrm{x})$ is the remainder term of Taylor's formula. If $R_{n}(x)=a+b x$ is satisfied, then $\mathbf{f}(\mathbf{x})$ is stable. Including generalized functions $\mathbf{f}(\mathbf{x})$.

The polar coordinate system indicates: the increment of the function $y=a$ tis liblar, so this function is stable.

Note: Because it is a steady increment, the conclusion is established.

\section{The principle of storing DNA bases and RNA bases}

The bases in DNA and RNA have the function of storing electric energy. Electricity generated by the gravitational waves of the earth and the sun is stored on the base, which is equivalent to capacitance. The difference of potential between different bases is related to molecular weight (electronic number).[3,7]

The potential level is first: base $\mathrm{C}$ and base $\mathrm{U}$.

Potential level second: base T.

Potential level third: base A.

Potential level 4.5: base G.

\section{Bridge properties of hydrogen bonds}

When the hydrogen group forms hydrogen bonds (base pairs), it has the function of bridge. Maintain relative potential difference. Hydrogen bonds form the charging function under the action of the earth gravitational wave field and the solar gravitational wave field. The relative potential level is increased by one level, that is, the relative differential rise level. When the hydrogen bond is cut off, it returns to its original state, and the potential difference is reduced to one level.

\section{The number of base pairs in DNA}

If the gravitational wave field is stable, the wave element satisfies the stability equation.

The spiral of

$$
y=a+b \theta
$$

The two wave sources (earth and sun):

Two relative rotation wave sources $\mathrm{N}_{1}$ and $\mathrm{N}_{2}$, relatively stable states (two rotation axes are not parallel), and the stability equations are:

$$
y_{1}=a_{1}+b_{1} \theta_{1}, y_{2}=a_{2}+b_{2} \theta_{2}
$$

So, The track is spring oval.

$P$ is any point on the elliptic path, and $F_{1}, F^{F}$ is the two focus on the long axis of ellipse.

According to the definition of ellipse, 


$$
y=\left|F_{1} P\right|+\left|F_{2} P\right|=y_{1}+y_{2}=a_{1}+a_{2}+b_{1} \theta_{1}+b_{2} \theta_{2}
$$

is a constant.

The function ${ }^{y}$ is linear when and only when

$$
\theta_{1}=\theta_{2}=2 k \pi \text { or } \theta_{1}=\theta_{2}=2 k \pi+\pi, k=1,2,3 \ldots \text {, }
$$

is used. According to the stability theorem, the helical elliptic equation of solar gravitational wave is stable only if

$$
\theta_{1}=\theta_{2}=2 k \pi \text { or } \theta_{1}=\theta_{2}=2 k \pi+\pi, k=1,2,3 \ldots .
$$

There are only two pairs of DNA base pairs.

\section{v. DNA base pairs and DNA information}

Properties of base pairs:

Base pairs have directivity.

- The difference of a base pair is a 2 stage potential difference, and the difference of the other base pair is a 3.5 stage potential difference.

- The state of base pairs in the energy field is Charging process, and the difference between the two base pairs should be increased by one level. The transcription process returned to its original level.

Directions: the base pairs in positive direction are A-T, G-C.

The reverse direction base pairs are T-A, C-G.

The base pair of small difference is A-T, T-A.

The base pair with large difference is $\mathrm{G}-\mathrm{C}, \mathrm{C}-\mathrm{G}$.

The base pair potential difference between $\mathrm{A}$ and $\mathrm{T}$ is 2 , and the base pair potential difference between $\mathrm{G}$ and $\mathrm{C}$ is 3.5 .

During the transcription process, two deoxyribonucleotide chains were decomposed, and the difference between A and T was 1 grade, which could not be combined. In order to maintain the 2 level difference, the combination of A and U in messenger RNA ensures 2 level differential. A small amount of A is combined with C.

- During transcription, the two deoxynucleotide chains break down, and the difference between $\mathrm{G}$ and $\mathrm{C}$ becomes 2.5 . In messenger RNA, the binding of $\mathrm{G}$ and $\mathrm{C}$ remains unchanged. But base $\mathrm{G}$ can also bind to $\mathrm{U}$. The difference between $\mathrm{G}$ and $\mathrm{U}$ is also 2.5 .

\section{vi. Conclusion}

The formation and storage principle of DNA information will play a promoting role in the field of DNA function research. The design field of Quaternary computer, the research on the stability of information received by quantum communication, and the research field of the stability of long-range electromagnetic laser beam will play a reference value.

\section{References}

1. Zhe Yin, “Zhe Yin's energy and wave theorem”. International Journal of Engineering and Advanced Research Technology, 2(3), 2016, 11-15. 
Proc. of the Seventh Intl. Conf. on Advances in Bio-Informatics, Bio-Technology and Environmental Engineering - ABBE 2018 Copyright $($ Institute of Research Engineers and Doctors, USA .All rights reserved.

ISBN: 978-1-63248-161-0 DOI: 10.15224/978-1-63248-161-0-12

2. Zhe Yin, “ The function and relation of proton and neutron”. International Journal of Recent Scientific Research,8(1), 2017,15321-15324.

3. Zhe Yin, "Effect of Gravitational Wave on DNA Base”. International Journal of Recent Scientific Research, 8(2), 2017,15705-15707.

4. Zhe Yin, "The formation of elements". IOSR Journal of Applied Physics,9(2), 2017,08-13.

5. Zhe Yin, Liu, D., “ Electron trajectory”. International Journal of Applied Science and Mathematics,3( 2), ,2016,56-57.

6. Zhe Yin, Liu, D., “Causes of sound wave on the moon”. International Journal of Engineering and Applied Sciences,3(2),2016, 56-58.

7. Zhe Yin, Zhang, W., Bie, H., “Zhe Yin's theorem of molecular biology”. Academia Journal of Scientific Research,4(4), 2016,99-102.

About Author (s):

Zhe Yin

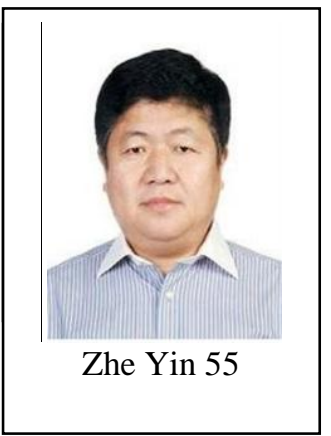

Bio Mathematics. Bio information. 